\title{
Power Savings from Half-Duplex Relaying in Downlink Cellular Systems
}

\author{
Chandrasekharan Raman, Gerard J. Foschini, Reinaldo A. Valenzuela, \\ Roy D. Yates and Narayan B. Mandayam
}

\begin{abstract}
We evaluate the peak and average power savings due to relay deployments in cellular systems via a simulation study. The peak power savings translate to cost reduction in power amplifiers. The average power savings lead to savings in electricity bills. Half-duplex relays are placed one per sector in a 19-cell, 57-sector cellular network. In the baseline case, the base stations control their transmit powers to achieve a common rate among users. When relays are present in the system, optimal powers are found when the relays get the complete message to be relayed to the user. The codebooks at the relays are chosen such that the users obtain a rate corresponding to the sum of the received powers from the base station and the relay. We observe that when power control is employed, the peak power saving is $2.6 \mathrm{~dB}$ and the average total power in the system can be reduced by $3 \mathrm{~dB}$.
\end{abstract}

\section{INTRODUCTION}

The deployment of relays in cellular system has recently been standardized in the WiMAX, IEEE 802.16j [1] and is an active topic of discussion in the advanced specifications of 3GPP-Long Term Evolution (LTE) [2]. Although commercial relay deployments in cellular systems are not prominent at present, it has been proposed that future systems with relays would operate in certain specific ways. For instance, the relays could be dedicated network elements, placed at certain locations (planned or unplanned) in the cell to help 'forwarding' the message from the base station to the user in the downlink, and from the user to the base station in the uplink. These devices could be smarter than ordinary repeaters and could perform some digital base band processing to improve reception at the destination terminals. Because they rely on air interfaces, relays avoid the back-haul costs involving data aggregation and infrastructure costs associated with backbone connectivity. However, there are a lot of open issues yet to be resolved. An important question of practical significance is the usefulness of deploying relays in an interference limited cellular network. In this work, we quantify the power savings due to relaying in cellular systems, assuming the availability of low-cost (cheaper than base stations) relaying devices with advanced signal processing capabilities. We impose a practical restriction that the relays are half-duplex. We envisage a scenario wherein the low-powered (with transmit powers of an order of magnitude lesser than base stations) relays placed

C. Raman, R. D. Yates and N. B. Mandayam are with WINLAB Rutgers University, North Brunswick NJ \{chandru, ryates, narayan\}@winlab. rutgers. edu

G. J. Foschini and R. A. Valenzuela are with the Wireless Communications Research Department, Bell Labs, Alcatel-Lucent, Holmdel NJ \{gjf, rav\}ealcatel-lucent. com on roof-tops, lamp-posts or building walls help in relaying the message over to the user locations.

Relaying in wireless networks has been an active area of research. From an information theoretic point of view, but for some coding strategies proposed by Cover and El Gamal in [3] for special cases of the single relay channel, the capacity of the general relay channel is still unknown [4]. Though most of the earlier works assume that the relay can transmit and listen over the same band, the half-duplex constraint, i.e., they cannot simultaneously transmit and receive in the same frequency band, is taken into account in later works, e.g., [5], [6]. The information theoretic studies reveal that when there are one or two relays, the best strategy is to optimally combine the source and relay transmissions at the user location, rather than multihopping from the source to the destination through the relay(s). The intuition is that the user can make use of signals from both the source and the relay to get a better signal strength and hence a better rate. Multihopping on the other hand, ignores the signal from the source, however strong it is.

The information theoretic relaying protocols mentioned above often involve complicated multiuser coding and decoding techniques, that are too far from being practically feasible. There have been some recent works trying to bridge the gap between the information theoretic and practical multihopping schemes, e.g., [7]-[9]. Most of the results in these works correspond to the case of linear network of nodes, where there is a single commodity flow of message from the source node to sink node through a set of relay nodes. The interference, if at all, is only due to simultaneous transmissions from different relay nodes. This can be completely eliminated, by multiuser coding/decoding techniques. Such analysis does not however, carry over directly to the cellular systems since there are multiple simultaneous flows and multiuser techniques may incur significant overhead.

There are few other works, e.g., [10]-[12] that evaluate the gains due to relay deployments in cellular systems. Our work differs from these in that we propose a power control scheme and a transmission scheme wherein the base stations and the relays collaborate to help the user in getting the message. The transmit powers in this collaborative scheme are optimized for power savings. Power control in cellular systems [13] has been well studied in the context of both uplink and downlink systems. While there exists distributed power control algorithms [14], transmit power control for cellular networks in the presence of relays has not been considered to the best of our knowledge. In this paper, we propose a framework 


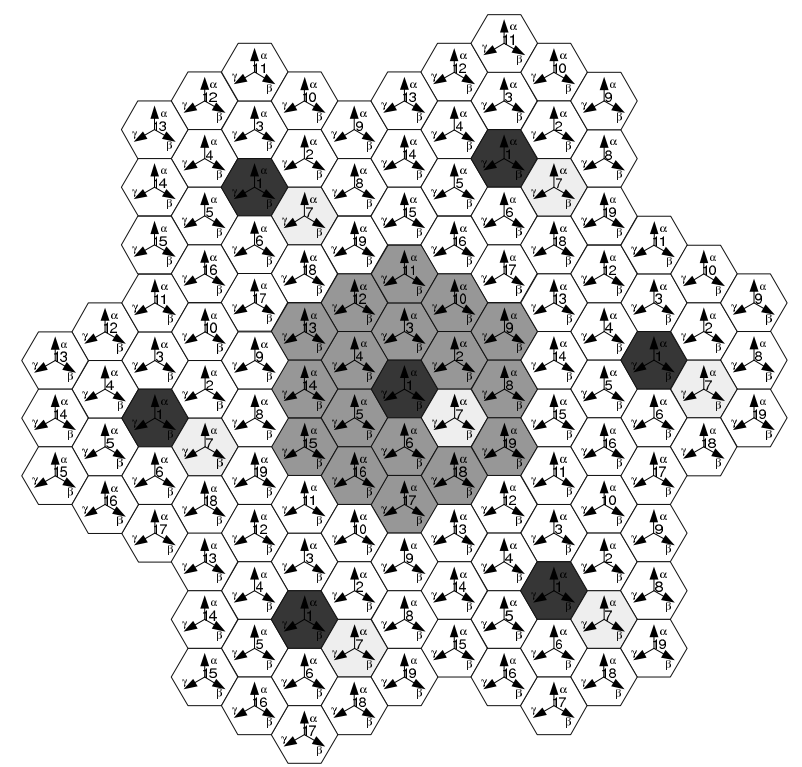

Fig. 1. Wrap-around simulation model. The center ring of 19 cells are used for the simulation. The surrounding cell activity is mirrored in the center ring. The direction of the arrows represent the direction of the main lobe of the sectorized antenna.

for joint power control of base station and relay powers in the downlink. The relays, due to the proximity to the user locations, substitute for the base station powers to maintain the same desired rate. This translates to power savings for the base stations.

This paper is organized as follows. Section II explains the simulation set-up and parameters of the simulation model. Section III explains the details on how power control is performed in the baseline case as well as the case when relays are present in the system. Simulation results are presented in Section IV and we conclude with some discussions in Section V.

\section{SYSTEM DESCRIPTION}

We evaluate the power savings in the base station locations due to relay deployments in a cellular system. However, to model and simulate all the dynamics of a cellular system can be too complicated. To overcome such difficulties, we make some reasonable simplifying assumptions and take an idealized look at the model and operation of a cellular system in our work. The assumptions are consistent across systems with and without relays to make a fair comparison. We consider a cellular system with idealized hexagonal cells with a base station at the center of each cell. The topology is shown in Figure 1. The first two tiers of interferers are considered and the activities of the farther tier of cells are mirrored by the center ring of 19 cells. The site-to-site distance (distance between any two base stations) is taken to be 1 mile. The cells are divided into $120^{\circ}$ sectors, each sector illuminated by a base station antenna pattern given by

$$
A(\theta)=-\min \left(12\left(\frac{\theta}{\theta_{3 d B}}\right)^{2}, A_{\max }\right),
$$

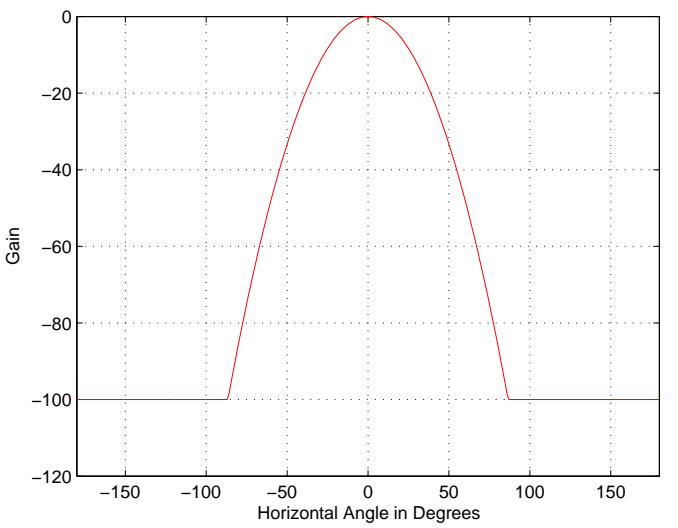

Fig. 2. Antenna gain pattern (from [15]) as a function of the horizontal angle in degrees. The mathematical expression for the gain is given in Equation (1).

where $A(\theta)$ is the antenna gain in $\mathrm{dBi}$ in the direction $\theta$, $-180^{\circ} \leq \theta \leq 180^{\circ}$, $\min ($.$) denotes the minimum function,$ $\theta_{3 d B}$ is the $3 \mathrm{~dB}$ beamwidth and $A_{\max }=20 \mathrm{~dB}$ is the maximum attenuation. The antenna gain pattern is shown in the Figure 2.

At the receiving terminal (relay or user), the transmitted power undergoes attenuation due to the distance traveled and shadowing effects around the receiver. The propagation attenuation between a transmitting terminal (base station or relay) and a receiving terminal (relay or user) consists of the path loss and the shadowing component. We assume path loss with a 3.8 propagation exponent and log-normal shadowing with mean $0 \mathrm{~dB}$ and a standard deviation of $8 \mathrm{~dB}$. The shadowing is assumed to be spatially uncorrelated and fixed for a given set of user locations. The base station and the relay antenna gains are taken to be $15 \mathrm{~dB}$ (at zero degree horizontal angle) and user antenna gain as $-1 \mathrm{~dB}$. Other losses account for $10 \mathrm{~dB}$. Together with the above losses, we include the antenna pattern loss to calculate the received power. The receiver noise figure is set at $5 \mathrm{~dB}$, and the thermal noise power at the each receiving terminal (relay or user) is assumed to be $-102 \mathrm{dBm}$. The effect of small scale fading is ignored in our simulations.

All users share the same band of frequencies and hence simultaneous transmissions interfere with each other. The total interference at each receiving terminal from all transmitters in the system is modeled as Gaussian noise and the achievable rate to a user $i$ at time $t$ is calculated as the Shannon rate $r_{i}(t)=\log _{2}\left(1+\operatorname{SINR}_{i}(t)\right)$, where SINR denotes the signalto-interference plus noise ratio.

\section{A. Coding Strategy}

We assume transmission using a certain class of Gaussian codebooks termed as universal codes [16]. Whenever the mutual information between the base station and relay terminal is better, the relays can decode the message with a shorter block length. The relay then transmits the same message as the base station is transmitting using a predetermined codebook 
so that the mutual information adds up at the user location. Similar coding strategies have been considered in [17], [18]. In the former, co-located users jointly relay the message to the destination, when each of them decode the source before the destination. In [18], the authors propose a the dynamic decode and forwarding methodology, where the relays start transmitting immediately after they are decode the source. This ensures that the relays are helpful as soon as they are eligible to help the source to deliver the message to the destination.

\section{B. Placement of users and relays in the system}

Continuing with our idealization with the set-up, we operate the system such that only one user per sector is active at any time slot or frequency band. Hence, in a 19-cell network with 3 sectors per base antenna, at most 57 users can be served at one resource unit, i.e., time slot or frequency band in an Orthogonal Frequency Division Multiple Access (OFDMA) system. In our simulations, we randomly place users uniformly across the network one-by-one until all 57 base station sectors are occupied. Each user associates himself with the base station with the highest received signal strength. If the base station sector is already occupied by another user, the user is not allowed into the system and a new user realization is generated. The users are equipped with an omni-directional antenna.

The benefits of relays e.g., power savings and throughput improvements depend on the interference generated by the relays, which in turn, depends on the transmit power, geographic location of the relays and the propagation environment. We experiment with various relay placements and the simulation results are presented for the relay locations for which the gains are found to be maximum. We do not necessarily place the relays at optimal locations. A relay with an omni-directional antenna is placed at half the cell radius in the direction of the main lobe of each base station sector antenna. The relays are associated with the corresponding base station sector. The relay powers are also varied so as to improve the peak power savings.

\section{POWER CONTROL WITH RELAYS IN DOWNLINK CELLULAR SYSTEM}

In this section, we propose a framework for power control in the downlink of a cellular system with relays. The baseline system for comparison is the conventional cellular downlink power control scheme wherein the base stations adjust their powers to achieve a target rate for all users. We let $10 \%$ of the whole population of users to be in outage, i.e., they are not guaranteed any non-zero rate. The need for allowing $10 \%$ outage is to avoid dragging down the performance of the served users under our strict constraint of serving these users with guaranteed minimum rate. Otherwise, the mutual interference between users could drag the guaranteed minimum rate down to zero in the limit of large networks. We then fix the target rate to be the same for the system with relays. When relays are present in the system, peak power required at the base stations to achieve the same target rate is lesser. This reduction leads to peak power savings. The proximity of relays to the user locations leads to savings in average power in the system.

\section{A. Operating the baseline system}

In the baseline case, each base station sector powers down its transmitted power within the peak power limitations so that $90 \%$ of users are guaranteed $r_{0} \mathrm{bits} / \mathrm{sec} / \mathrm{Hz}$. Since all users share the same band of frequencies, they observe interference from all the base station sectors in the system. We use a common subscript for a base station or a user in a particular sector. If at time $t, p_{i}(t)$ is the power of the transmitting base antenna corresponding to the $i$ th user and $h_{i j}$ is the channel gain, including path loss and shadowing, from the $j$ th base to the $i$ th user and $\sigma^{2}$ is the noise power at the receiver, the instantaneous SINR (signal-to-interference-plus-noise ratio) of the $i$ th user in the system is given by

$$
\rho_{i}(t)=\frac{h_{i i} p_{i}(t)}{\sum_{j \neq i} h_{i j} p_{j}(t)+\sigma^{2}} .
$$

We assume transmission using Gaussian codebooks. Thus, the corresponding instantaneous rate for the user $i$ is given by $r_{i}(t)=\log _{2}\left(1+\rho_{i}(t)\right) \mathrm{bits} / \mathrm{sec} / \mathrm{Hz}$. The set of feasible powers such that the users not in outage are guaranteed with a rate $r_{0}$ is obtained by solving for the feasibility of instantaneous rates subject to peak power constraints, specified by

$$
\begin{aligned}
\log _{2}\left(1+\rho_{i}(t)\right) & \geq r_{0}, \\
\text { i.e., } \rho_{i}(t) & \geq 2^{r_{0}}-1, \\
\text { subject to } p_{i}(t) & \leq p_{i, \max },
\end{aligned}
$$

for all users $i$ not in outage. In practice, each base station increases its power autonomously in small increments, until it hits the peak power limit or when the user associated with it attains the desired rate $r_{0}$. Users that make the power constraint to go active before attaining the desired rate are discarded.

In our simulations, we eliminate $10 \%$ of users (over a large number of user realizations) in the following way. From a user population of 57 users, we discard the user who caused the power constraint in (5) go active first. This reduces the interference coupled with other users. Within the remaining set of users, we discard the next user causing the power constraint to go active and continue in this fashion until all users are guaranteed a rate of $r_{0} \mathrm{bit} / \mathrm{sec} / \mathrm{Hz}$, by still meeting the peak power constraints. We remark here that the order in which the users are discarded results in different power levels from the base stations, due to variations in the interference coupling among the users. Hence depending on the peak powers limitations at the base stations, the order in which the users are dismissed should be chosen carefully. In our simulations, we assume similar peak power constraints for all base stations and relays across the network and we choose an arbitrary of order for discarding the users.

\section{B. Operating the system with relays}

When relays are present in the system, $10 \%$ of users are discarded in the manner similar to the baseline system. At 
time $t=0$, the relays do not have complete message required to relay to the user. Hence, the system starts out as it does for the baseline case. The base stations increase their powers autonomously in small increments targetting the users rates to increase. Users that do not meet the peak power constraints in (5) are eliminated one after the other. The remaining users get the desired rate without violating the peak power constraint at the base stations. The relay in each sector also listens to the transmission by the base stations. Depending on the channel conditions and coupling of interference from the adjacent sectors, each relay gets the message at different points in time. As and when the relay in the sector decodes the message from the base station, the relay helps the base station by transmitting a Gaussian code book such that the SINR as seen by the user from the relay and the base station adds up at the user location. The code books at the base stations and relays are designed such their received powers add up at the user location. In order to maintain the common desired rate for all users, the relay and base station jointly adjust their powers so that the user gets the desired rate. This ensures that the base station and relay transmit just enough power to the user to obtain the desired rate.

Let $p_{i}(t)$ denote the power transmitted by the base station sector $i$ at time $t$ and $h_{i j}$ be the channel gain, including path loss and shadowing, from the $j$ th base to the $i$ th user. Let $q_{i}(t)$ be the power transmitted from the relay $i$ at time $t$ and $g_{i j}$ be the channel gain to the user $i$ from the relay $j$. Then, when the relay and base station transmit simultaneously, effective SINR at $i^{t h}$ user location when the relay is active is given by

$$
\rho_{i}^{\text {relay }}(t)=\frac{h_{i i} p_{i}(t)+g_{i i} q_{i}(t)}{\sum_{j \neq i} h_{i j} p_{j}(t)+g_{i j} q_{j}(t)+\sigma^{2}} .
$$

As with the baseline case, the set of feasible powers (for both base station antennas and relays) such that the users not in outage are guaranteed with a rate $r_{0}$ is obtained by solving for the feasibility of instantaneous rates subject to peak power constraints

$$
\begin{aligned}
\log _{2}\left(1+\rho_{i}^{\text {relay }}(t)\right) & \geq r_{0} \\
\text { i.e., } \rho_{i}^{\text {relay }}(t) & \geq 2^{r_{0}}-1, \\
\text { subject to } p_{i}(t) & \leq p_{i, \max }, \\
\text { and } q_{i}(t) & \leq q_{i, \max },
\end{aligned}
$$

for all users $i$ not in outage. If we consider the transmit powers of the base stations and relays as variables of optimization, we have a total of $2 N$ variables, for $N$ base station sectors in the system. Thus, power control in cellular systems in the presence of relays gives us additional $N$ degrees of freedom to optimize over. The transmit powers in the system can be optimized to reduce the maximum peak power transmission in the system, reduce total energy in the system etc. In what follows, we assume that a "genie" has the knowledge of the all the channel gains between the base stations as well as relays and the users. We explain ways to achieve various aforementioned objectives using linear program (LP) formulations.

\section{Minimizing the total instantaneous transmit powers in the system}

In this section, we are interested in evaluating the benefits of relays in minimizing the total instantaneous sum power in the system while delivering a target common rate with $10 \%$ of the users being omitted from the system. The practical benefit of minimizing the total sum of transmit powers in a cellular system to save the energy costs in the network. Saving energy costs translate to saving electricity bills at the cell sites for the cellular service provider.

The desired common rate for the users is fixed at $r_{0}$ bits/symbol. We define $\mathcal{A}(t)$ as the set of all active relays at time $t$, i.e., the set of relays that have obtained the message and are ready to help the base station. $\mathcal{A}^{c}(t)$ denotes the complementary set of all inactive relays.

At a given time $t$, the "genie" solves the following optimization problem:

$$
\begin{array}{ccc}
\min _{\substack{p_{1}(t), \ldots, p_{N}(t) \\
q_{1}(t), \ldots, q_{N}(t)}} & \sum_{i} p_{i}(t)+q_{i}(t) \\
\text { subject to } & \log _{2}\left(1+\frac{h_{i i} p_{i}(t)+g_{i i} q_{i}(t)}{\sum_{j \neq i} h_{i j} p_{j}(t)+g_{i j} q_{j}(t)+\sigma^{2}}\right) \\
& \quad \geq r_{0}, i=1, \ldots, N,
\end{array}
$$

The above optimization problem (11) is an LP, since we can write the constraint (11b) as

$\frac{1}{2^{r_{0}}-1}\left(h_{i i} p_{i}(t)+g_{i i} q_{i}(t)\right)-\sum_{j \neq i}\left(h_{i j} p_{j}(t)+g_{i j} q_{j}(t)\right) \geq \sigma^{2}$,

for $i=1, \ldots, N$. Writing the equation in vector form, we have

$$
\begin{aligned}
s^{*}(t)=\min _{\mathbf{p}(t), \mathbf{q}(t)} & \mathbf{1}^{T}(\mathbf{p}(t)+\mathbf{q}(t)) \\
\text { subject to } & \mathbf{A p}(t)+\mathbf{B q}(t) \leq-\sigma^{2} \mathbf{1} \\
& \mathbf{0} \leq \mathbf{p}(t) \leq \mathbf{p}_{\max }, \\
& \mathbf{0} \leq \mathbf{q}(t) \leq \mathbf{q}_{\max } .
\end{aligned}
$$

where,

$$
\begin{aligned}
\mathbf{A} & =\left(\begin{array}{cccc}
-\frac{h_{11}}{2^{r_{0}}-1} & h_{12} & \cdots & h_{1 N} \\
h_{21} & -\frac{h_{22}}{2^{r_{0}}-1} & \cdots & h_{2 N} \\
\vdots & \vdots & \ddots & \vdots \\
h_{N 1} & h_{N 2} & \cdots & -\frac{h_{N N}}{2^{r_{0}-1}}
\end{array}\right), \\
\mathbf{B} & =\left(\begin{array}{cccc}
-\frac{g_{11}}{2^{r_{0}}-1} & g_{12} & \cdots & g_{1 N} \\
g_{21} & -\frac{g_{22}}{2^{r_{0}}-1} & \cdots & g_{2 N} \\
\vdots & \vdots & \ddots & \vdots \\
g_{N 1} & g_{N 2} & \cdots & -\frac{g_{N N}}{2^{r_{0}-1}}
\end{array}\right),
\end{aligned}
$$


and

$$
\begin{aligned}
\mathbf{p}(t) & =\left[p_{1}(t) \ldots p_{N}(t)\right]^{T}, \\
\mathbf{q}(t) & =\left[q_{1}(t) \ldots q_{N}(t)\right]^{T}, \\
\mathbf{p}_{\max } & =\left[p_{1, \max } \ldots p_{N, \max }\right]^{T}, \\
\mathbf{q}_{\max } & =\left[q_{1, \max } \ldots q_{N, \max }\right]^{T},
\end{aligned}
$$

where, $q_{i, \max }=0, i \in \mathcal{A}^{c}(t)$. Solution to the above LP provides the optimal power values that minimize the instantaneous total power in the system. We take a myopic approach of minimizing the total sum power of the system at time $t$ in order to reduce the total average power transmission in the system. Each time a relay becomes eligible for transmission, the LP is solved to find the best set of powers by minimizing the instantaneous powers in the system. Note that in some cases, when a relay is eligible to help the base station, turning off the base station may be the optimal thing to do. This choice comes out as a solution to the optimization program.

\section{Minimizing the peak transmit power}

Minimizing the peak transmit power leads to peak power savings in the system. A practical benefit of peak power savings is the huge savings in the cost of power amplifiers for the cellular base stations. If by deploying low-power relays in the system, we save in the cost of the power amplifiers of the base stations, cellular operators could save in capital expenses. To this end, the "genie" solves the following optimization problem of minimizing the maximum instantaneous transmit powers at the base stations:

$$
\begin{array}{ccc}
\min _{\substack{p_{1}(t), \ldots, p_{N}(t) \\
q_{1}(t), \ldots, q_{N}(t)}} & \max _{i} p_{i}(t) \\
\text { subject to } & \log _{2}\left(1+\frac{h_{i i} p_{i}(t)+g_{i i} q_{i}(t)}{\sum_{j \neq i} h_{i j} p_{j}(t)+g_{i j} q_{j}(t)+\sigma^{2}}\right) \\
& \quad \geq r_{0}, i=1, \ldots, N, \\
& 0 \leq p_{i}(t) \leq p_{i, \max }, i=1, \ldots, N, \\
& 0 \leq q_{i}(t) \leq q_{i, \max }, i \in \mathcal{A}(t), \\
& q_{i}(t)=0, i \in \mathcal{A}^{c}(t) .
\end{array}
$$

Rewriting the above LP in vector form yields,

$$
\begin{aligned}
p^{*}(t)=\min _{\mathbf{p}(t), \mathbf{q}(t)} & \alpha \\
\text { subject to } & \mathbf{A p}(t)+\mathbf{B} \mathbf{q}(t) \leq-\sigma^{2} \mathbf{1} \\
& \mathbf{0} \leq \mathbf{p}(t) \leq \mathbf{p}_{\max }, \\
& \mathbf{0} \leq \mathbf{q}(t) \leq \mathbf{q}_{\max }, \\
& \alpha \mathbf{1} \geq \mathbf{p}_{\max },
\end{aligned}
$$

where, A and $\mathbf{B}$ are given by (14) and (15) respectively.

\section{E. Increasing the common rate}

As a corollary to the above approaches, if we keep the peak power constant across both the baseline and the system with relays, we can increase the common targeted rate in the system with relays. The problem of maximizing the common rate can be posed as an optimization program with the transmit powers of the base station and relays as the variables. The "genie" then solves the optimization program:

$$
\begin{array}{ccc}
\max _{p_{1}(t), \ldots, p_{N}(t)} & r_{0} \\
\text { subject to } & \log _{2}\left(1+\frac{h_{i i} p_{i}(t)+g_{i i} q_{i}(t)}{\sum_{j \neq i} h_{i j} p_{j}(t)+g_{i j} q_{j}(t)+\sigma^{2}}\right) \\
& \geq r_{0}, i=1, \ldots, N, & (19 \mathrm{~b}) \\
& 0 \leq p_{i}(t) \leq p_{i, \max }, i=1, \ldots, N, & \text { (19c) } \\
& 0 \leq q_{i}(t) \leq q_{i, \max }, i \in \mathcal{A}(t), \\
& q_{i}(t)=0, i \in \mathcal{A}^{c}(t) .
\end{array}
$$

The optimization program above is a non-convex problem because of constraint set is non-convex. We solve this program by an iterative approach. We start with a low easily achievable target rate $r_{0}$ so that the constraint set (19b)-(19e) is feasible. We increase the target rate in small increments until the constraint set becomes infeasible. In each step, we get a set of feasible power assignments. The last set of feasible power assignments is the solution to the optimization program. The method converges, since the iterations generate a bounded sequence of increasing rates.

\section{Simulation Results}

We simulated a cellular network using the set-up mentioned in Section II. First, we evaluate the peak power savings. We operate the baseline system as well as the system with relays such that, over a large number of user loading iterations, $90 \%$ of users obtain a common desired rate of $1 \mathrm{bit} / \mathrm{sec} / \mathrm{Hz}$. We follow the iterative heuristic approach to discard users in the system. For the baseline case, at the outset, we solve (17) with $q_{i, \max }=0, i=1, \ldots, N$. The users that cause the power constraint in (17c) active are discarded one-byone. We stop when there exists a feasible set of powers $p_{1}, \ldots, p_{N}$. This way, for a given user loading in the network, we retain only those users that can obtain the desired common rate. The optimal value of (17) is the peak base transmit power required to deliver the common rate of $1 \mathrm{bit} / \mathrm{sec} / \mathrm{Hz}$ for the remaining $90 \%$ users in each instantiation of the user placements. We calculate the minimum peak base power required to deliver the common rate of $1 \mathrm{bit} / \mathrm{sec} / \mathrm{Hz}$ at $10 \%$ outage as the maximum of the peak power required among the large number of user loadings. By choosing appropriate values of $p_{i, \max }=p_{\max }$, we make sure that $10 \%$ of users are discarded over large number of user instantiations. An important observation was that the peak base power is limited by the propagation characteristics of the worst user that has not been discarded.

When relays are present in the system, we follow a similar iterative heuristic approach to discard users. We start by solving (17) and discard users that cause the power constraint in (17c) one-by-one. As time $t$ progresses, some relays become eligible to help the base stations. Each time a relay is eligible to help the base station, LP (17) is solved with updated $q_{i, \max }$ to find the new set of optimal powers. In our simulations, we slot time into 1000 mini-slots and at the end of each mini-slot, 
TABLE I

SUMMARY OF PEAK POWER SAVINGS DUE TO DEPLOYMENT OF RELAYS IN CELLULAR SYSTEMS

\begin{tabular}{|c|c|c|}
\hline \multicolumn{3}{|c|}{$\begin{array}{l}\text { Scenario: } 57 \text { sectors, one relay per sector, one user per sector. Base station and relays transmit just enough power } \\
\text { to maintain uniform rate using power control. }\end{array}$} \\
\hline $\begin{array}{c}\text { Peak power required to guarantee } \\
1 \mathrm{bit} / \mathrm{sec} / \mathrm{Hz} \text { at } 10 \% \text { outage } \\
\text { Base station only (No relays) }\end{array}$ & $\begin{array}{c}\text { Peak power required to guarantee } \\
1 \mathrm{bit} / \mathrm{sec} / \mathrm{Hz} \text { at } 10 \% \text { outage } \\
\text { Base station with relays }\end{array}$ & Savings in $\mathrm{dB}$ \\
\hline $10 \mathrm{~W}$ & $5.5 \mathrm{~W}$ & 2.6 \\
\hline $\begin{array}{l}\text { Common rate for } 90 \% \text { users } \\
\text { Base station only (No relays) }\end{array}$ & $\begin{array}{l}\text { Common rate for } 90 \% \text { users } \\
\text { Base station with relays }\end{array}$ & Percentage rate increase \\
\hline $1 \mathrm{bps} / \mathrm{Hz}$ & $1.35 \mathrm{bps} / \mathrm{Hz}$ & $35 \%$ \\
\hline
\end{tabular}

we check the eligibility of the each relay. If at the end of a mini-slot, a particular relay has received the complete message, the LP is solved to find the new set of powers. We stop when there exists a feasible set of powers $p_{1}, \ldots, p_{N}, q_{1}, \ldots, q_{N}$, to deliver the desired common rate for users not in outage in each instantiation. As with the baseline case, the minimum peak base power required to deliver the desired rate of $1 \mathrm{bit} / \mathrm{sec} / \mathrm{Hz}$ at $10 \%$-outage is the maximum of the peak powers required among user loadings. We observed that the peak power savings in the downlink when power control is employed were around $2.6 \mathrm{~dB}$.

To evaluate the average power savings, we solve (11) to minimize the total power in the system. For the same target rate of $1 \mathrm{bit} / \mathrm{sec} / \mathrm{Hz}$ for both the baseline system and the system with relays, we observed that the average power savings were $3 \mathrm{~dB}$. When the peak power constraints were fixed for the baseline system and the system with relays and the optimization program in (19) is solved, we observe that the there is $35 \%$ improvement in the throughput for $90 \%$ users in the system, with the baseline system being served at 1 $\mathrm{bit} / \mathrm{sec} / \mathrm{Hz}$.

\section{CONCLusions}

We simulated the downlink of cellular system wherein a common rate of $1 \mathrm{bps} / \mathrm{Hz}$ is achieved by $90 \%$ users in the system. We proposed a framework for power control in a downlink cellular system when relays are present in the system. The framework can be posed as a linear program formulation when the relays help the user by power addition at the user locations. This formulation can be used to evaluate the average and peak power savings in the system. The peak power savings are $2.6 \mathrm{~dB}$ and average power savings are upto $3 \mathrm{~dB}$. We also see a $35 \%$ improvement in the common rate for fixed peak power levels at the base station. The results are summarized in the Table I.

This study is not exhaustive and there are interesting issues that opened up during the course of this study. One of them was to exploit the half-duplex nature of the relays to perform smart resource allocation. Half-duplex relays cannot simultaneously transmit and receive at the same time and frequency band. We could exploit this feature so that we schedule the base station and relay transmissions at the appropriate time slots such that there are rate improvements over the baseline case. Another important issue is to find practical ways to achieve the collaborative power addition schemes.

\section{REFERENCES}

[1] S. W. Peters and R. W. Heath, "The future of WiMAX: Multihop relaying with ieee 802.16j," IEEE Commun. Magazine, vol. 47, pp. 104111, January 2009.

[2] 3GPP TR 36.913 v. 8.0.0, "Requirements for Further Advancements for E-UTRA (LTE-Advanced)," June 2008.

[3] T. M. Cover and A. A. E. Gamal, "Capacity theorems for the relay channel," IEEE Trans. Inf. Theory, vol. 25, pp. 572-584, Sept 1979.

[4] G. Kramer, M. Gastpar, and P. Gupta, "Cooperative strategies and capacity theorems for relay networks," IEEE Trans. Inf. Theory, vol. 51, pp. 3037-3063, Sept 2005.

[5] M. A. Khojastepour, A. Sabharwal, and B. Aazhang, "On the capacity of 'cheap' relay networks," in Proc. 37th CISS, 2003. Baltimore, MD.

[6] G. Kramer, "Models and theory for relay channels with receive constraints," in Proc. Allerton Conf. on Commun., Control, and Comp.,, 2004. UIUC, IL.

[7] D. Chen, M. Haenggi, and J. N. Laneman, "Distributed spectrumefficient routing algorithms in wireless networks," IEEE Trans. Wireless Commun., vol. 7, pp. 5297-5305, Dec 2008.

[8] M. Sikora, J. N. Laneman, M. Haenggi, J. D. J. Costello, and T. E. Fuja, "Bandwidth- and power-efficient routing in linear wireless networks," IEEE Trans. Inf. Theory, vol. 52, pp. 2624-2633, June 2006.

[9] C. Raman, R. Yates, and N. Mandayam, "Cross layer scheduling of endto-end flows using a spectrum server," in Proc. CISS, 2006. Princeton, NJ.

[10] H. Viswanathan and S. Mukherjee, "Performance of cellular networks with relays and centralized scheduling," IEEE Trans. Wireless Commun., vol. 4, pp. 2318-2328, Sept 2005.

[11] O. Oyman, "Oppurtunistic scheduling and spectrum reuse in relay-based cellular OFDMA networks," in Proc. Globecom, 2007. Washington DC.

[12] K. Balachandran, J. Kang, K. Karakayali, and J. Singh, "Capacity benefits of relays with in-band backhauling in cellular networks," in Proc. ICC, 2008. Beijing, China.

[13] J. Zander, "Distributed cochannel interference control in cellular radio systems," IEEE Trans. Veh. Technol., vol. 41, pp. 305-311, Aug 1992.

[14] G. Foschini and Z. Miljanic, "A simple distributed autonomous power control algorithm and its convergence," IEEE Trans. Veh. Technol., vol. 42, pp. 641-646, Nov 1993.

[15] "Multi-hop relay system evaluation methodology." http://ieee802.org/16.

[16] D. Blackwell, L. Breiman, and A. J. Thomasian, "Capacity of a class of channels," Annals of Mathematical Statistics, vol. 30, pp. 1229-1241, 1968.

[17] M. Katz and S. Shamai, "Transmitting to colocated users in wireless ad hoc and sensor networks," IEEE Trans. Inf. Theory, vol. 51, pp. 35403563 , Oct 2005 .

[18] K. Azarian, H. E. Gamal, and P. Schniter, "On the achievable diversityvs-multiplexing tradeoff in half-duplex cooperative channels," IEEE Trans. Inf. Theory, vol. 51, pp. 4152-4172, Dec 2005. 